\author{
Gdańsk 2018, Nr. 39 \\ https://doi.org/10.26881/sgg.2018.39.04
}

Ruxandra Cosma

Universität Bukarest

\title{
Von Sachverhalt, Tatsache und propositionalen Pro-Elementen: Ein Blick über das Deutsche und Rumänische
}

In honorem Ulrich Engel

\begin{abstract}
Vorliegender Aufsatz schlägt ein Gerüst für das Inventar propositionaler Pro-Formen der deutschen und rumänischen Sprache vor. Als Bausteine werden auf Form-Ebene sachverhaltsbezeichnende ProFormen das und asta genommen. Die semantische Interpretation der Pro-Formen ist komponential, kontext-abhängig und weist das und asta nebst anderen Bedeutungskomponenten deskriptive Bedeutung zu. Nach diesem Muster können auch das/asta-einbettende Ausdrücke interpretiert werden. Ferner werden Ähnlichkeiten zwischen den diskutierten Pro-Elementen und propositionalen Nomina herausgestellt, was die Möglichkeit bietet, letztere semantisch i. als nominale Korrelate und ii. versehen mit verweisender Funktion zu betrachten.
\end{abstract}

Schlüsselwörter: Pro-Formen, Tatsache, das, asta

On Sachverhalt, Tatsache and propositional proforms: a view of German and Romanian. The paper proposes a simplified architecture for the inventory of propositional proforms of the two contrasted languages, German and Romanian. Basic elements of the system are the demonstratives das/asta, which represent the foundation for more complex ones, specifically for the so-called German pronominal adverbs and for the asta-embedding prepositional phrases in Romanian. The semantic interpretation of the proforms is componential, context-dependent and adds descriptive meaning to das and asta, therefore also to proforms embedding them. The paper then describes proforms in relation to content nouns, which offers the possibility of semantically interpreting these i. as nominal correlates, and ii. in their anaphoric function.

Keywords: propositional proforms, Tatsache, das, asta

\section{Hintergrund}

Der vorliegende Aufsatz ist vorerst nicht der Betrachtung prädikativer Lexeme gewidmet, die eine Sachverhaltsbeschreibung einleiten und die Relation des Betrachters zu dem Inhalt Objektsatzes ausdrücken (Einstellungsprädikate): als semantisches Argument nehmen diese eine Proposition, die syntaktisch unterschiedlich realisiert wird - durch ein satzförmiges Komplement (einen dass-Satz im Deutschen), ein propositionales Nomen/container noun wie z.B. Tatsache ('Gewissheitsprädikat' bei Reis 1997: 203), durch eine indirekte Frage 
(ob-Satz im Deutschen), einen Infinitivsatz etc. Solche Einstellungsprädikate ${ }^{1}$ drücken mentale Zustände aus, Geisteshaltungen, die man gegenüber Propositionen haben kann, wie etwa Überzeugung (mentaler Zustand), Denken (mentaler Prozess), Wissen (epistemischer Zustand), Absicht, Wunsch (mentale Disposition), Behauptung, Zweifel (Sprechhandlung), Freude (Emotion) etc. Auch Ontologie und Gebrauch von Konzepten wie 'Sachverhalt', 'Ereignis,' 'Situation' sind nicht Gegenstand des Aufsatzes; Unterscheidungskriterien für Propositionen auf Ebene der (philosophischen) Semantik sind umstritten und uneinheitlich. Noch befasst sich der Aufsatz mit der im Diskurs geteilten Information und deren Aktualisierung. Der Aufsatz konzentriert sich auf sachverhaltskondensierende Pro-Elemente, die semantisch und distributionell, in Relation zueinander und sprachvergleichend beschrieben werden, in dem Versuch, ein Muster in dem Bestand propositionaler Pro-Formen zu identifizieren.

Das Ziel der Betrachtung ist, zu zeigen,

i. dass die Bedeutung propositionaler Pro-Formen komponential ist und dass diese kontextabhängig unterschiedlichen Beitrag leisten,

ii. dass die Klasse von Pro-Elementen an sich eine Architektur aufweist,

iii. dass ein propositionales Pro-Element wie das deskriptive Bedeutung hat und ein propositionales Nomen wie Tatsache verweist.

Es ist in der Tradition der von Ulrich Engel (ein-)geleiteten Projekte zum Valenzlexikon deutsch-rumänisch (Engel / Savin 1983) und der Kontrastiven Grammatik Deutsch-Rumänisch (Engel / Isbășescu / Stănescu / Nicolae 1992), dass hier zum Sprachpaar Deutsch-Rumänisch ein Beitrag geliefert wird. Dieser möchte zeigen, dass durch den Sprachvergleich auch andere Interpretationsmöglichkeiten für das Deutsche berücksichtigt werden können. Die Frage, ob eine propositionale Pro-Form an sich einen semantischen Wert hat oder als "Spur" (vgl. Breindl 2013) verstanden werden sollte (vgl. auch Frey / Meinunger / Schwabe 2016), ist dabei zentral.

Vorerst ein Beispiel für eines dieser Pro-Elemente, in Übersetzung:

(1) a. Man achtete darauf, dass niemand ohne Einladungskarte den Saal betrat (Engel 1996:258)

b. S-a avut grijă de asta, ca nimeni să nu intre fără invitație în sală.

Mit Blick auf Strategien der semantischen Argumentdoppelung (vgl. Cosma \& Engelberg 2014) im Satz selber und satzübergreifend, wenn Korrelatverbindungen vorliegen, sowie mit Blick auf die eigene semantische Kraft dieser Pro-Elemente werden mehrere Wege (in) der Versprachlichung des Sachverhalts unterschieden. Der Sachverhalt an sich, wenn er durch den Objektsatz beschrieben wird, vgl. hierzu u.a. Hole (2014: 28), wird bestimmt durch a. Weltbezug, b. Zeitbezug, c. den Bezug auf sachverhaltsbeteiligte Referenten und d. durch Wahrheitsbedingungen des prädikativen Lexems, das die Sachverhaltsbeschreibung einleitet.

Folgende terminologische Differenzierung möchte vereinfachend zeigen, dass propositionale Pro-Formen Bedeutungsmerkmale und Funktionen bündeln, die kontextabhängig aktualisiert bzw. wiederhergestellt werden. Unterschieden wird zunächst zwischen

1 Einstellungsverben sind Verben wie glauben, vermuten, hoffen, meinen, leugnen, bezweifeln, bestätigen, sich freuen, lieben, wissen, erfahren, fragen, nachforschen, untersuchen etc. 
i. einem Sachverhaltsausdruck, der zentral über Propositionen erfolgt,

ii. dem Ausdruck des Sachverhalts, der Einordnung des Sachverhaltes, dem Verweis auf den Sachverhalt über content nouns (propositionale Nomina); diese leisten in der Gruppe ihrerseits unterschiedlichen semantischen Beitrag,

iii. der Sachverhaltsnennung und dem kontextabhängig stärkeren oder schwächeren Verweis auf den Sachverhalt über zentrale Pro-Elemente das/asta,

iv. einer inkorporierten Sachverhaltsnennung und dem Verweis auf den Sachverhalt über Pronominaladverbien, und

v. der Sachverhaltsbeschreibung durch den Sprecher, der kommunikativen Gewichtung (in) der Proposition. In diesem Sinne wird die Relation zwischen Pro-Form und Sachverhalt im Deutschen an der Schnittstelle von Syntax, Pragmatik, Prosodie und Semantik diskutiert (u.a. Breindl 2013, Frey / Meinunger / Schwabe 2016).

Ein Sachverhaltsausdruck erfolgt zentral über Propositionen; entsprechende grammatische Einheit ist der Satz oder der Infinitivanschluss. Weitere Varianten sind auch Parataxe-/Nebenanordnungs-Situationen (Ich will mein Abonnament kündigen. Ist das per e-Mail möglich??2), de re Konstruktionen (vom Typ Peter glaubt von seiner Nachbarin, dass sie Harry Potter geschrieben hat), indirekte Rede-Instanzen mit Konjunktiv, oder direkte Rede-Instanzen.

Als Aussage über die Welt sind Propositionen wahr oder falsch, der Sachverhalt selbst ist in der möglichen Welt vorhanden oder nicht vorhanden ${ }^{3}$. Eine Definition für Proposition wird hier aus der Engel-Grammatik (1996: 71; 879) zitiert:

„Jede Äußerung beschreibt einen Sachverhalt oder bezieht sich auf einen solchen; dies ist ihr Inhalt. Aber zugleich drückt jede Äußerung - und darin geht es über den Satz hinaus - eine bestimmte Sprechhandlungsintention aus."

So ist die Proposition die Bedeutung einer Äußerung unter Ausschluss der Illokution, der Redeabsicht.

Für den kondensierten Sachverhaltsausdruck im Rumänischen gilt als Basisform asta 'das' (feminine singular pronoun with neutral value, Pană Dindelegan 2012, Pană Dindelegan ed. 2013), die auch in PP-en eingebettet wird: despre asta [darüber], la asta [daran] etc. ${ }^{4}$; das Wortbildungsverfahren Komposition ist im Rumänischen im Unterschied zum Deutschen nicht produktiv. Ob die zusammengesetzte Form der Pronominaladverbien ähnlich im Deutschen analysiert werden kann oder hingegen interpretiert wird, dass das Deutsche eine andere Strategie entwickelt hat und auf ein anderes Morphem zurückgreift, wie die Struktur des Pronominaladverbs da(r)-Präposition in den meisten Grammatiken des Deutschen beschrieben wird, auf diese Frage möchte der vorliegende Aufsatz aufmerksam machen und das zur Basiseinheit vorschlagen.

Daniel Glattauer, Gut gegen Nordwind. Goldmann. 2006/2008. S. 6

Vgl. u.a. Pafel\&Reich (2016: 12): „Eine falsche Proposition [..] kann nicht mit einer Situation in der Außenwelt identifiziert werden"

4 Giurgea (2012), Pană Dindelegan (2012) bringen eine sprachgeschichtliche und regionale Betrachtung des pronominalen Demonstrativsystems. Eine Beschreibung des Inventars oder eines Teilinventars sachverhaltsausdrückender Pronomina bieten Zafiu (2004), Pană Dindelegan (2012), eine Untersuchung von asta im gesprochenen Rumänisch bietet Nicula (u.a. 2008). 
Das Rumänische besitzt wie auch andere Sprachen kein Wort, das dem Sachverhalt ganzheitlich entsprechen würde; kontextabhängig wird der Sachverhalt durch faptul 'Tatsache', circumstanțe 'Umstände', häufig durch situație 'Situation', chestie 'Chose, Ding, Sache', treabă 'Sache, Ding, Arbeit' wiedergegeben. Kontrastive Arbeiten zum hier gewählten Sprachpaar sind Lăzărescu (1980), der u.a. die Beschreibung verbgesteuerter Korrelate im deutsch-rumänischen Verbvalenzlexikon (Engel / Savin 1982) bespricht, in gewisser Hinsicht auch Lupșan / Marki (2013) zur Distribution und Übersetzung der deutschen Form das im Rumänischen. Nicht zuletzt sei hier die KGDR (Engel et al. 1993) genannt.

\section{2. $\mathrm{Zu}$ das und Tatsache}

Die eine These des vorliegenden Ansatzes ist, dass propositionale Pro-Ausdrücke, in der Grammatikschreibung beschrieben als Funktionswörter, mehr semantisch gefüllt sind als soweit angenommen. Pro-Formen sind zunächst 'gleichwertig' und 'gleichgeltend' mit satzförmigen Komplementen (Zifonun / Hoffmann / Strecker 1997:37), die auf einen Sachverhalt referieren. Durch die Bestimmung als Funktionswörter werden sie generell mit einer beschränkten deskriptiven Bedeutung beschrieben.

Der Ansicht, dass diese beschränkte deskriptive Bedeutung aufweisen, kann aber an das und asta entgegenhalten werden. In Anlehnung an Löbner (2003: 30-31) werden zunächst einige terminologische Klärungen und Beschreibungen angeführt, die die Bedeutung von Sätzen und von Nomina/Inhaltswörtern bestimmen:

- Die deskriptive Bedeutung eines Satzes ist seine Proposition; dies ist ein Konzept für seine potenziellen Referenzsituationen, für eine bestimmte Art von Situation. Die Referenzsituation enthält die Referenten aller referierenden Elemente des Satzes in einer bestimmten Konstellation (Löbner 2003: 30).

- Die deskriptive Bedeutung eines Inhaltswortes ist ein Konzept für seine potenziellen Referenten; z.B. im Falle propositionaler Nomina (Tatsache, Gedanke, Idee etc.) handelt es sich um Referenz auf abstrakte Objekte, Ereignisse, Umstände etc. (Löbner 2003: 31). Ein Ausdruck hat folglich deskriptive Bedeutung, wenn er eine mentale Repräsentation ausdrückt, d.h. wenn er denotiert.

Die Annahme ist hier, dass das und rum. asta, als zentrale Kategorien für den pronominal zusammengefassten Sachverhalt in den beiden Sprachen, auch Ausdrucksbedeutung aufweisen, i.e. auch außerhalb eines Kontextes, in eigenem Sinne den Sachverhalt benennen, denotieren können. Ausdrucksbedeutung ist im Sinne von Löbner (2003) die Bedeutung eines einfachen oder zusammengesetzten Ausdrucks für sich genommen. Dies würde bedeuten, dass das/asta, dann auch komplexere Pro-Formen, die das/asta miteinschließen oder heranziehen, die Fähigkeit besitzen, den Sachverhalt anzugeben bzw. zu benennen, bevor er durch den Rest der Korrelatverbindung, durch den Kontext expliziert wird. Mit anderen Worten ist es die Annahme, dass die Funktion der Basiselemente das/asta nicht nur auf phorischen oder deiktischen Verweis reduziert werden sollte, sondern dass zugleich die Fähigkeit, mentale Repräsentationen, Bilder in unserem Kopf, zu aktivieren, besteht. Die Ausdrucksbedeutung ist in Situationen besser erkennbar, in denen die Verweiskraft von das/asta sehr schwach oder 
vage ist, in denen das 'Antezendensfeld' (Marx 2011:10) zu groß ist und der Sachverhalt selbst kaum rekonstruiert werden kann.

Pronominaladverbien hingegen sind nicht wie das mono-morphematisch, daher sind sie semantisch kompositionell zu betrachten. Komponenten ihrer Bedeutung sind die Bedeutungen der Teile, die Beziehung, in der diese Teile zueinander stehen, die deiktische oder phorische Verweiskraft, ihre kontextabhängige (explikative) Füllung. Nun hängt es in hohem Maße davon ab, wie die Teile semantisch interpretiert werden.

Propositionale Nomina, die propositionalen Pro-Formen in Bedeutung und Funktion ähnlich sind, haben als Nomina zunächst einmal „Benennungsfunktion“ (Engel 2004: 286). In der Reihe propositionaler Nomina sind sie inhaltlich sehr verschieden, Tatsache/rum. faptul wird jedoch in verschiedenen Sprachen am häufigsten als Korrelat identifiziert. Eine Klassifikation zur Syntax dieser Nomina, die dass-Sätze, $o b$-Sätze, Infinitivkonstruktionen selegieren, bieten u.a. Zifonun et al. (1997: 1978-1979):

I. Annahme, Glaube, Möglichkeit, Vermutung, Wunsch, Erinnerung etc. mit nicht-faktiv interpretierbaren dass-Sätzen,

II. Bedauern, Beleg, Beweis, Grund, Hinweis, Tatsache, Zeichen etc., mit faktiv interpretierbaren dass-Sätzen,

III. Antwort, Auskunft, Aussage, Behauptung, Einwand etc., die Indirektheitskontexte erzeugend sind,

sowie Alternationen u.a. bei Frage, Kontrolle, Zweifel, die ob-Sätze und $w$-Fragesätze als Nomenkomplemente nehmen; bei Glaube, Möglichkeit, Vertrauen, Wunsch, Zeichen, Antwort, Behauptung, die auch Infinitivkonstruktionen wählen.

Propositionale Nomina, die in der Literatur nach sehr unterschiedlichen Kriterien klassifiziert und benannt werden, wie container nouns bei Vendler (1967), der facts von events unterscheidet, content nouns bei Moulton (2009), shell nouns - von Schmid (2000) generell als konzeptuelle Hüllen für Informationsblöcke beschrieben, werden vorliegend nur in der Differenzierungsfunktion nach semantischen Typen verstanden. Für diese Klasse gibt es weitere Namen wie unspezifische Nomina (unspecific nouns), metasprachliche Nomina (metalanguage nouns) bei Winter (1992), anaphorische Nomina (A-nouns) bei Francis (1986), satzbezogene Verweisformen bei Koeppel (1993), Träger-Nomina (carrier nouns) bei Ivanič (1991) etc. Die Definition von Francis (1986:2) von A-Nomina als linguistische Wegweiser (signalposts), die dem Leser signalisieren, dass bestimmte Informationen an anderer Stelle im Text gefunden werden können, kann hier heraus gewählt werden, um Ähnlichkeit mit das und anderen propositionalen Pro-Formen herauszustellen. Geschieht der Ausdruck über satzförmige Prädikate in einer Argumentbeziehung, so handelt es sich um eine "explikative Satz-Erweiterung" (Fabricius-Hansen / von Stechow 1989). Bedeutung und Funktionen von shell nouns sind ihrerseits komponentiell: diese binden ein (contain), signalisieren (signal), zeigen hin (point), umhüllen (encapsulate) (vgl. Kolhatkar / Hirst 2014: 499).

Eine Klassifikation von shell-nouns kann hier in Anlehnung an Schmidt (2000: 4) illustriert werden. Solche Nomina sind in ihrer Bedeutung

- faktisch:fact, thing, point, problem, reason, difference etc.

- sprachbezogen: news, message, rumour, report, proposal etc.

- mental: idea, notion, belief, assumption, aim, plan etc. 
- modal: possibility, truth, permission, obligation, need etc.

- ereignisbeschreibend: act, move, measure, reaction, attempt, tradition etc.

- umstandsbeschreibend: situation, context, place, area, time, way etc.

Das Verhältnis von Sachverhalt und Tatsache, hier diesmal für sich betrachtet, wird vereinfachend zusammengefasst: wird ein Sachverhalt, als Gegenstand einer Aussage, überprüft und stellt sich dieser als wahr heraus, so wird er zu einer wahren Aussage; stellt sich dieser aber als falsch heraus, dann handle es sich nicht um einen Sachverhalt.

In dem vorliegenden Aufsatz, der nur auf die modulare Struktur in dem Bestand der Pro-Formen und auf eine komponentiale Betrachtung aufmerksam machen möchte, wird der Sachverhalt als übergreifend verstanden. Tatsache und weitere propositionale Nomina sind hingegen types, Hüllen für unterschiedliche Typen von Ereignissen und Propositionen (vgl. Schmid 2000). Ein Nomen wie Tatsache trägt dementsprechend den Sachverhalt in sich. Nach Kolhatkar / Zinsmeister / Hirst (2013: 300) charakterisiert fact/Tatsache und beschriftet/benennt (im Sinne von labeln) zugleich dessen Antezedens.

In Auswahl werden einige solche 'Hüllen' in Anlehnung an Schmid (2000: 78) präsentiert, wobei in Klammern die structure-inherent gaps, die von den Nomina eröffneten Leerstellen, angegeben werden. Die inhaltliche Bedeutung wird diesen vorangehend angeführt:

$\begin{array}{ll}\text { fact } & \text { state of affairs (true) } \\ \text { intention } & \text { state of wanting to do (activity) } \\ \text { way } & \text { manner, method of (event, activity) } \\ \text { attempt } & \text { act of trying to do (activity) } \\ \text { remark } & \text { (content of) act of saying something relevant. }\end{array}$

Zusammenfassend: abstrahiere man von der Verwendung der Ausdrücke in konkreten Kontexten, so könne das allgemeine Potential der Ausdrücke bestimmt werden. Es ist die These hier, dass die Frage nach dem allgemeinen Potential von das im Vergleich mit dem Ausdruckspotential von propositionalen Nomen betrachtet werden kann, so wie die Äußerungsbedeutung von propositionalen Nomina und von Pro-Formen aus dem gegebenen Äußerungskontext resultiert.

Aus der Rezeptionsperspektive hat das unterschiedliche Verweiskraft. Laut Marx (2011: 10) motivieren solch komplex-anaphorische Ausdrücke die Generierung eines neuen, komplexen (abstrakten) Referenten: „Komplexe Referenten müssen aktiv konstruktiert und als Einheit einer Repräsentation der Textbedeutung etabliert werden." Um dies in die vorliegende Annahme einzubetten: Der abstrakte Referent ist zwar neu, weil er ein jedes Mal in einem Kontext neu entsteht, wird aber durch die Pro-Form wie durch das Nomen jeweils als Sachverhalt und/oder als Typ charakterisiert.

Weiter unten ein Textbeispiel mit unterschiedlich indizierbaren Interpretationsmöglichkeiten:

(2) [Mia hat übrigens gefragt, [ob ich nicht mitkommen will $\left.]_{\mathrm{i}}\right]_{\mathrm{i}}$. Ich habe geantwortet: $[\mathrm{Das}]_{\mathrm{i}}$ ist IHM aber ganz bestimmt nicht Recht. ${ }^{5}$

5 Daniel Glattauer, Gut gegen Nordwind. Roman. 2008, 13. Auflage. Goldmann. S. 116. 
Auch in den weiteren das-Instanzen kann der Sachverhalt nicht abgegrenzt werden; er ist über eine Summe von Ereignissen, Situationen, Umständen, Eigenschaften etc. beschreibbar:

(3) Das ist Berlin ${ }^{6} \quad$ (Hymne von Berlin der Band Endlich August)

(4) Das ist Netzpolitik! $\quad$ (als Konferenztitel)

Für (3) stellt das Rumänische drei Varianten zur Verfügung; es ist wohl das Bedürfnis, die Unbestimmtheit aufzulösen. Dies geschieht u.a. über einen Wechsel von der sachverhaltsbezeichnenden femininen Form asta auf ăsta (mask./neutr. Demonstrativum 'dieser/ dieses'), welches hinzeigend viel stärker wirkt. Eine daraus sekundär resultierende Lesart ist 'So ist Berlin' (Așa e Berlinul). Hier kann ferner angeknüpft werden an die an sich berechtigte Mitberücksichtigung des „fakultativen Korrelats“ felul 'Art' im Rumänischen, wie in Lăzărescu (1980:152) z.B. für (6) diskutiert wird.

(5) Asta e Berlinul. Așa e Berlinul. 'So ist Berlin'

(6) $\mathrm{Eu}$

Ich

voi păstra mereu în amintire

cum

Ss behalten immer

m-ai

ajutat.

wie CLT.AKK.1SG.-haben.PRÄs.2SG geholfen.

Ich werde $[\mathrm{es} / \mathrm{das}]_{\mathrm{i}}$ immer in Erinnerung behalten, [wie du mir geholfen hast $]_{\mathrm{i}}{ }^{\prime}$

Eine Rekonstruktion 'stromaufwärts' (Brandom 2001; im Sinne inferentieller Semantik ${ }^{8}$ ) ist im nächsten Beispiel möglich:

(7) „Ziemlich groß“, sagt sie, während sie sich in der Suite umblickt. „Sechs Zimmer für einen Mann. Willst du mir nicht was zu trinken anbieten?“ „Möchtest du...?“ „Nein, danke.“ Sie gibt der Zimmerkontrolle die Anweisung, Musik zu spielen. Mozart. „Aber eigentlich magst du gar keine Musik, nicht wahr?"“ „Nicht diese Art. Das ist mir zu ... bieder."

Aus der Perspektive der oben genannten Annahme lassen sich Dativus iudicantis-Konstruktionen mit das betrachten. Diese setzen Grenzen; ein „Vergleichsmaßstab ist [...] durch etwas vorgegeben, das gut für den Dativreferenten ist und von ihm auch als Zweck verfolgt wird“ (Hole 2013: 6):

6 Endlich August: "CSD, Charité/ Rummelsburg an der Spree/Insel ohne Meer,/tausend Seen/ Kennzeichen B./Flieger brauch ich nicht /für Venedig oder Bangkok/Auch Istanbul ist drin,/ wenn ich mich auf mein Rad hock/Nächte sind wie Tage hier,/ nur ein bisschen dunkler/Wir lieben die Freiheit/ doch spielen im Käfig Fußball und Ping Pong/New York ist King,/ was ist Berlin?/ Natürlich King Kong/Egal ob Atze oder Göre/ Lady oder Gentleman/Jedem Topf sein Deckel/ und Töpfchen auf sein Deckelchen/Ja, das ist Berlin"

7 https://netzpolitik.org/13np/

8 Brandom (2001: 250): „Die behauptbaren Gehalte, die von Aussagesätzen ausgedrückt werden, deren Äußerung diese Art von Kraft besitzen kann, müssen dementsprechend in beiden normativen Dimensionen inferentiell gegliedert sein. Sie müssen, sozusagen stromabwärts, inferentielle Konsequenzen haben, wobei sich die Festlegung auf diese Konsequenzen infolge der Festlegung auf den ursprünglichen Gehalt ergibt. Und sie müssen, stromaufwärts betrachtet, inferentielle Vorgänger haben, also Beziehungen zu Gehalten, die als Prämissen fungieren können, von denen die Berechtigung zum ursprünglichen Gehalt geerbt werden kann.“”

9 Pierce Brown, Red Rising - Im Haus der Feinde. Üb. Von Bernhard Kempen. Heyne 2016. 
(8) Das ist mir zu früh/zu viel/zu schnell/zu langsam

Der Sachverhalt, auf den mit iudicantis-Konstruktionen Bezug genommen wird, ist meist unscharf. Die Pro-Form identifiziert zunächst die Kategorie Sachverhalt, ruft das abstrakte, mentale Bild für den Sachverhalt ab; dann erst folgt der Versuch, den Sachverhalt wiederherzustellen. Hier zufälligerweise ein kataphorischer Verweis:

(9) Kolumne: Das ist mir zu kompliziert

„Einfach wollen wir sein. Glaube, und du wirst gerettet / Die schwierigen Fragen zu stellen, das tut uns Frommen weniger gut an. / Vielleicht noch weniger, auf Antworten zu warten und um sie zu ringen. / Zweifeln ja, doch lieber an der Kirche, bevorzugt an den anderen. / Den iPad können wir bedienen, doch wehe es kommt eine Frage, auf die es keine Standardantwort gibt! / Einfach haben wir es uns gemacht. / Der Zweck heiligt die Mittel. / Funktioniert's, ist es erlaubt. / Stimuliert's, bekommt es den Stempel ,approved“. / Alles ist gut, was nicht über 20 Minuten dauert. / Kulturell sind wir abgehängt, die Quittung für unsere „me too“-Strategie [...].“10

Über asta-Dativus iudicantis-Konstruktionen verfügt das Rumänische nicht:

$\begin{array}{lllll}\text { (10) }{ }^{*} \text { Asta } & \text { mi-e } & \text { prea } & \text { mult } & \text { 'Das ist mir zu viel.' } \\ \text { Das } & \text { CLT.DAT.1sG.-sein.3sG } & \text { zu } & \text { viel }\end{array}$

Die These, dass Pro-Formen komponential beschreibbar sind, knüpft u.a. an Nunberg (1993) oder an Zifonun (2001) an. Laut Nunberg (1993) kodieren persönliche und demonstrative Pronomina drei Komponenten:

(i.) eine deiktische Komponente, die als deskriptive Information verstanden werden kann, durch die auf ein außersprachliches Objekt etc. als Index verwiesen wird,

(ii.) eine klassifikatorische Komponente, die leitet die Interpretation des Ganzen an und bringt z.B. $\phi$-Merkmale wie Genus oder Belebtheit (animacy),

(iii) eine relationale Komponente, die die Beziehung zwischen dem Objekt, welches von der deiktischen Komponente bestimmt wird, das ist der Index, und der intendierten Interpretation beschreibt.

Ähnlich werden Teilaufgaben für Pronomina von Zifonun (2001: 9-11) identifiziert: „sie (Pronomina) erfüllen durch Deixis, Phorik, Quantifikation, Interrogation oder Selektion wesentliche Teilaufgaben, die von den entsprechenden Inhaltswörtern selbst nicht erfüllt werden könne.“

Weiter unten wird ein Überblick über die wichtigsten propositionalen Pro-Elemente beider Sprachen gebracht, seien diese nun als Substitute von Sachverhalten (propositionale Pro-Formen) oder als pronominale Kopien von Sachverhalten, bei Paranhos Zitterbart (2013: 602) Bezugselemente im komplexen Bezugsausdruck, stellvertretend oder verstärkend im Matrixsatz für ein sententiales oder infinites Komplement eingesetzt. In dem Bestand lässt sich wie in der Semantik dieser Pro-Formen tentativ ein moduläres Muster interpretieren. 3. Propositionale Pro-Formen im Deutschen und im Rumänischen

10 http://hanniel.ch/2018/04/13/kolumne-das-ist-mir-zu-kompliziert/ (28.05.2018). 
Vorgeschlagen wird hier, dass das Inventar der propositionalen Pro-Elemente im Deutschen das, im Rumänischen asta als Basiseinheit nimmt. Zum Deutschen u.a.

i. $\quad e s$ in Subjekt- oder Objektfunktion:

(11) $[\text { Es }]_{i}$ wundert mich, [dass ich nichts von ihr höre $]_{i}$. Er hat $[e s]_{i}$ leider aufgegeben, [die Situation zu klären $]_{i}$.

ii. abstrakte Demonstrativa wie das und dies,

(12) Ich habe dir $[\mathrm{das}]_{\mathrm{i}}$ doch so oft erklärt, [dass hier Verkehrszeichen nicht beachtet werden $]_{\mathrm{i}}$

iii. dessen, dem

(13) Man sagt, [die Zeit heile alle Wunden]. [Dem] stimme ich nicht gerade $z u$.

iv. Präpositionaladverbien wie daran, darauf, daraus, dabei, dadurch, dafür, dagegen, darin, damit etc., die in Alternation das Muster mit deiktischem bier-nehmen, oder auch interrogativ $w o-(\mathrm{r}-)$ in Kombination mit einer relativ großen Anzahl von Präpositionen (vgl. u.a. IDSGrammatik 1997: 1475) gebildet werden.

(14) Ich glaube [daran],.. [dass Falten um deine Augen und deinen Mund zeigen, dass du im Leben viel gelacht hast. $]_{i}^{11}$

v. das Relativpronomen was in Spaltsätzen (Pseudo-clefts) ${ }^{12}$

(15) $[\text { Was }]_{i}$ mich überrascht ist, [dass ich jeden Abend von ihnen höre $]_{i}$.

Während die beiden genitivischen und dativischen Formen zu das gehören, haben deutsche Pronominaladverbien als komponierte Formen eine erkennbare interne Struktur mit einem „deiktischen“ Teil oder mit einem Demonstrativpronomen $;{ }^{13}$ die Form sollte zur Rekonstruktion der kommunikativen Rolle verhelfen (vgl. Rehbein 1995). Einerseits ist das in konkurrierenden Versprachlichungsstrategien für Pronominaladverbien formenthalten: darüber - über das, davon - von dem, - ̈̈ber was etc., in der interrogativen Variante was: worauf - auf was, worüber - über was, wofür - für was etc. Andererseits, interpretiert man das so, dass auf den Sachverhalt über Lokaladverbien da- und hier-verwiesen wird, so wird der Sachverhaltsausdruck durch eine "lokaldeiktische Verweispotenz" (Graefen 2009: 697) gestützt. Bei Hermann Paul (1919, 3: 155) ist ein Hinweis zu lesen, dass „das Adverb da ursprünglich als Kasusvertretung nur mit Bezug auf einen Satz, auf einen Gedanken angewendet wurde.“ Ferner schreibt Paul (1919, Bd. 3: 154):

„Die enge Beziehung zwischen Pron. und pronominalem Adv. zeigt sich besonders in einer Eigenheit, die den westgerm. Dialekten gemein ist. Statt einer Präp. mit Dat. oder Akk. der Pronomina das und was wird eine Verbindung der dazu gehörigen Ortsadverbien mit dem der Präp. entsprechenden Adverbium verwendet, also z.B. danach statt nach dem."

Dies würde bedeuten, dass der Sachverhalt im Pronominaladverb historisch drin war; die Wandlung zum Bezug auf den Sachverhalt ist anreichernd und zugleich abschwächend, wählt man die Interpretation mit deiktischem Element $d a$ -

Pronominaladverbien, von Eroms (1981: 267) definiert als „stark komprimierte Syntagmen, die mit verbenthaltenden Syntagmen (Infinitivkonstruktionen, Ergänzungssätzen)

11 www.bento.de, 17.05.2018: Pink wird von Typ wegen ihres Alters beleidigt - und reagiert perfekt $(20.05 .2018)$

12 Pseudo-Clefts

13 https://grammis.ids-mannheim.de/systematische-grammatik/523 
kommutieren", lassen sich syntaktisch wie PP-en interpretieren. Die Präposition fungiere als Kopf mit einem nominalen Komplement, oder anders ausgedrückt, als "eine gesättigte Präposition" (Geuder 2014: 13). ${ }^{14}$ Von der Struktur her sind auch solche mit Konnektoren-Status (Adverbkonnektoren) wie deswegen, stattdessen, trotzdem, deshalb, dementsprechend etc. zu berücksichtigen. Die Verschmelzung beider Komponenten des Pronominaladverbs, wie immer diese syntaktisch auch analysiert wird (vgl. Gallmann $1997 \mathrm{zu} w$-Wörtern), ist im Sinne von Stiebels (2002) eine weitere Strategie der sprachlichen Ökonomie für das Argumentlinking.

Die Rolle von asta ist im Rumänischen fassbar. Das Rumänische besitzt keine formale Entsprechung für es. Ein thematisches es in Objektposition wird wie im Deutschen durch das/rum. asta realisiert, oder gar nicht. In diesem Zusammenhang sei für das Rumänische auf die Null-Anapher (u.a. Pană Dingelegan 2013: 588) aufmerksam gemacht:
(16) Stiam [pro/asta]! 'Ich wusste $e s ! '$
wissen.PRÄT.1SG. [pro/das]
Dem $e s$ in Subjektposition entspricht im Rumänischen nur eine Null-Anapher:
(17) $[\text { Es }]_{\mathrm{i}}$ freut mich, [dass du anrufst $]_{\mathrm{i}}$

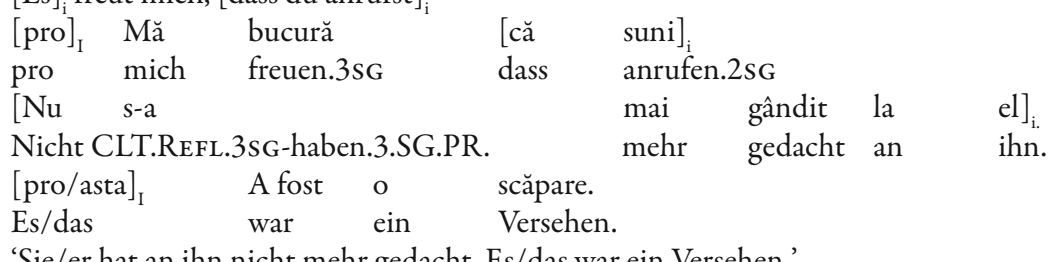
'Sie/er hat an ihn nicht mehr gedacht. Es/das war ein Versehen.'

Zum abstrakten Demonstrativum in femininer und proximaler Kurzform asta 'das' muss hinzugefügt werden, dass es außerdem noch eine Langform (aceasta) gibt, während eine distale wie aceeal aia 'jenes' häufig für nicht-argumentalen (kausalen, finalen) Sachverhaltsbezug genommen wird: de aceea 'deswegen', pentru aceea/aceasta 'deshalb, dafür' etc. Die Präferenz für Kurz- und Langform ist registersensitiv, asta hat sich jedoch in Objektposition durchgesetzt.

Im Unterschied zum Deutschen besteht die Möglichkeit, kumulativ Sachverhalte zu bezeichnen: astea/acestea (vgl. 20, 21). Die Pluralform astea ist durch multe 'viele', toate 'alle' modifizierbar.

(19) Și toate astea unde duc? ? $^{15}$ 'Und wohin führt all dies?' Und alle diese wohin führen

(20) Nu contează neapărat să câștigi 10 mii de euro pe lună, contează să stai propriul tău șef, să simți libertatea aia [..], multe astea care în codul muncii intră la demnitate în muncă ${ }^{16}$

'Man braucht nicht 10.000 Euro im Monat zu gewinnen, es heißt vielmehr, dass man sein eigener Chef bleiben kann, diese Freiheit verspürt [..], all das was in dem Arbeitsgesetzbuch unter Arbeitswürde fällt.'

14 Geuder (2014: 13): „Einen weiteren verwandten Fall stellen die sog. Pronominaladverbien dar, wo traditionell eine Klassifikation als Adverb vorgenommen wird, weil das Wort als ganzes gesättigt ist: da.mit / her. auf / wo.für."

15 https://sufletdinboabedecafea.wordpress.com/2018/05/08/si-unde-toate-astea-duc/

16 https://opencube.ro/prezent-si-viitor/(15.05.2018) 
Dem deutschen Pronominaladverb der Form da-(r)-Präposition entspricht im Rumänischen eine asta- einbettende PP (21): pentru asta 'für das/dafür', de asta 'von dem/davon', cu asta 'mit dem/damit' etc.. Pluralische Markierung ist auch in asta-enthaltenden PP-en möglich:

(21) Pentru asta nu există nicio scuză Für das nicht es gibt keine Entschuldigung 'Dafür gibt es keine Entschuldigung '/Es gibt keine Entschuldigung'

(22) Pentru astea și pentru multe altele, vă mulțumesc! Für das.PL.Fem. und für viele andere.DEF CLT.Pl.2 DAT danken.1sG. 'Für all dies und vieles mehr danke ich euch!'

In Objektposition wird asta über ein fem. Klitikon -o verdoppelt, das Klitikon kann auch selbständig vorkommen in (23b); in dem Fall hat es stärkere Verweiskraft. Es gibt auch Instanzen sprachlicher Überblendung, in denen der Sachverhalt innersentential verdoppelt, satzübergreifend mehrfach ausgedrückt wird (23c):

(23)

$\begin{array}{lll}\text { Asta am } & \text { înțeles-o } & \text { acum } \\ \text { Das } & \text { hab. Präs.3SG verstanden-Clt.Fem.SG.AKK. } & \text { jetzt } \\ \text { 'Das habe ich jetzt verstanden' } & \end{array}$
'Das habe ich jetzt verstanden'

b. A rezolvat-o haben.1.sG gelöst.-CLT.FEM.SG.AKK. 'Das habe ich gelöst.'

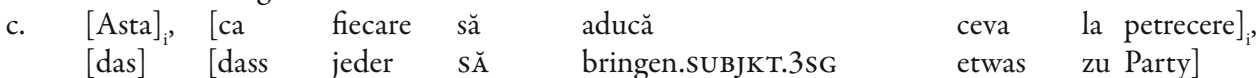
am înțeles-[o $]_{\mathrm{i}}$ deja haben.1sg verstanden-CLT.FEM.SG.AKK bereits. 'Das, dass jeder etwas zur Party mitbringen soll, das habe ich bereits verstanden'

Der vorliegende Aufsatz hat soweit versucht, den propositionalen Pro-Formen-Bestand in seiner Modularität, nach dem Baukastenprinzip zu betrachten. Dies bedeuteut nicht nur, dass die Bausteine auf Form-Ebene oder auf semantischer Ebene zusammengefügt, ineinander geschachtelt werden, gegeneinander ausgetauscht werden können, sondern auch dass sie häufig über Schnittstellen interagieren (vgl. das/asta und Tatsache/faptul, es in Alternation zu das, asta und -o etc.). In diesem Sinne seien abschließend Präferenzen für semantische Argumentverdoppelung im Satz und satzübergreifend genannt, wie in früheren Untersuchungen gezeigt wurde (Cosma \& Engelberg 2014: 378); bei inhaltlich komplexeren propositionalen Nomina sind es hingegen Argumentspaltungsstrategien. Argumentdoppelung liegt vor allem vor, wenn das syntaktische Argument stärker integriert werden soll (wie z.B. asta und -o im Rumänischen), semantische Argumentspaltung hingegen, wenn zwei oder mehrere Konstituenten mehr zur semantischen Spezifizierung des Arguments beitragen, wie hier durch das willkürlich gewählte propositionale Nomen Gedanke zum vorliegend vorgeschlagenen Gedankenspiel:

(24) Leichten Mutes parliert sie [über den Gedanken $]_{\mathrm{i}}$, [dass sich manches auch machen ließe”.... $]_{\mathrm{i}}^{17}$

17 In leichter Abwandlung DeReKo (cosmas IDS): Z00/012.03269 Die Zeit (Online-Ausgabe), 06.12.2000; Noch Fragen, Hauser? 


\section{Literatur}

Brandom, Robert B. (2001): Begründung und Begreifen: Eine Einführung in den Inferentialismus. Frankfurt: Suhrkamp.

Breindl, Eva (2013): Präpositionalobjektsätze. In: Meibauer, Jörg / Steinbach, Markus / Altmann, Hans (Hg.) (2013): Satztypen im Deutschen. Berlin / New York: de Gruyter, 458-481.

Cosma, Ruxandra, Stefan Engelberg (2014): Subjektsätze als alternative Valenzen im Deutschen und Rumänischen. Eine kontrastive, quantitative Korpusstudie zu Psych-Verben. In: Cosma, Ruxandra / Engelberg, Stefan / Schlotthauer, Susan / Stănescu, Speranța / Zifonun, Gisela (Hg.): Komplexe Argumentstrukturen. Kontrastive Untersuchungen zum Deutschen, Rumänischen und Englischen. Berlin: Akademie-Verlag, 339-420.

Duden (2016): Die Grammatik. Unentbehrlich für richtiges Deutsch. Bd. 4. 9., überarbeitete Auflage. hrsg. von Angelika Wöllstein und der Dudenredaktion. Berlin: Dudenverlag.

Engel, Ulrich / Savin, Emilia (1983): Valenzlexikon deutsch-rumänisch. (Deutsch im Kontrast 3, Institut für deutsche Sprache, Mannheim). Heidelberg: Julius Groos.

Engel, Ulrich / Isbășescu, Mihai / Stănescu, Speranța / Nicolae, Octavian (1992): Kontrastive Grammatik deutsch rumänisch (=KGDR). Heidelberg: Julius Groos.

Engel, Ulrich (1996): Deutsche Grammatik. 3., korrigierte Auflage. Heidelberg: Julius Groos.

Engel, Ulrich (2004): Deutsche Grammatik. Neubearbeitung. München: iudicium.

Eroms, Hans-Werner (1981): Valenz, Kasus und Präpositionen. Untersuchungen zur Syntax und Semantik präpositionaler Konstruktionen in der deutschen Gegenwartssprache. Heidelberg: Winter.

Fabricius-Hansen, Cathrine / von Stechow, Arnim (1989): Explikative und implikative Nominalerweiterungen im Deutschen. In: Zeitschrift für Sprachwissenschaft 8 (2), 173-205.

Francis, Gill (1986): Anaphoric nouns. Birmingham: English Language Research, University of Birmingham.

Frey, Werner/Meinunger, André / Schwabe, Kerstin (Hg.) (2016): Inner-sentential propositional proforms. Syntactic properties and interpretative effects. (Linguistik Aktuell 232). Amsterdam/Philadelphia: Benjamins.

Gallmann, Peter (1997): Zu Morphosyntax und Lexik der w-Wörter. (Arbeitspapiere des Sonderforschungsbereichs 340, Sprachtheoretische Grundlagen für die Computerlinguistik, Bericht 107). Tübingen: Universität Tübingen.

Geuder, Wilhelm (2014): Die Kategorie “Adverb” in einem hierarchischen Modell der Wortarten. Jahrestagung der Deutschen Gesellschaft für Sprachwissenschaft AG 7: Probleme der syntaktischen Kategorisierung Marburg, 5. März 2014. Online: http://home.uni-leipzig.de/doering/ AG7_PraesHandout/Geuder.pdf. (15.05.2018).

Giurgea, Ion (2012): Constituirea formelor scurte ale demonstrativelor în română. In: Zafiu, Rodica / Dragomirescu, Adina / Nicolae, Alexandru (Hg.): Limba română: direcții actuale în cercetarea lingvistică. Bucureşti: Editura Universităţii din Bucureşti, 129-138.

Graefen, Gabriele (2009): Pronomen. In: Hoffmann, Ludger (Hg.): Handbuch deutscher Wortarten . Berlin / New York: de Gruyter, 657-705.

Hole, Daniel (2014): Dativ, Bindung und Diathese. (Studia grammatica 78). Berlin / New York: de Gruyter.

Ivanič, Roz (1991): Nouns in search of a context. In: International Review of Applied Linguistics, XXIX (2), 93-114. 
Koeppel, Rolf (1993): Satzbezogene Verweisformen: eine datenbankgestützte Untersuchung zu ihrer Distribution und Funktion in mündlichen Texten, schriftichen Texten und schriftlichen Fachtexten des Deutschen. (Tübinger Beiträge zur Linguistik 386). Tübingen: Narr.

Kolhatkar, Varada / Zinsmeister, Heike / Hirst, Graeme (2013): Interpreting Anaphoric Shell Nouns using Antecedents of Cataphoric Shell Nouns as Training Data. In: Proceedings of the 2013 Conference on Empirical Methods in Natural Language Processing. Seattle, Washington, USA, 18-21 October 2013, 300-310.

Kolhatkar, Varada / Hirst, Graeme (2014): Resolving shell nouns. In: Proceedings of the 2014 Conference on Empirical Methods in Natural Language Processing (EMNLP). October 25-29. 2014. Doha, Qatar, 499-510.

Lăzărescu, Ioan (1980): Das Korrelat im Deutschen und im Rumänischen. In: Beiträge zur Deutsch-Rumänischen Kontrastiven Grammatik. Zweites Kolloquium des Kollektivs zur DRKG. Iași. 2-3. November 1979, 149-161.

Löbner, Sebastian (2003): Semantik. Eine Einführung. Berlin: Walter de Gruyter.

Lupșan, Karla/Marki, Marianne (2013): Zur Übersetzung der Form das ins Rumänische. In: Temeswarer Beiträge zur Germanistik 10, 221-246.

Marx, Konstanze (2011): Die Verarbeitung von Komplex-Anaphern. Neurolinguistische Untersuchungen zur kognitiven Textverstehenstheorie. Berlin: Universitätsverlag der TUB.

Moulton, Keir (2009): Natural Selection and the Syntax of Clausal Complementation. Diss. Amherst: University of Massachusetts.

Nicula, Irina (2008): Utilizări pragmatice ale demonstrativului în limba vorbită actuală: asta vs aceasta. In: Pană Dindelegan, Gabriela (Hg.): Limba română. Dinamica limbii, dinamica interpretării. Editura Universităţii din Bucureşti, 127-133.

Nunberg, Geoffrey (1993): Indexicality and deixis. In: Linguistics and Philosophy 16, 1-43.

Pafel, Jürgen / Reich, Ingo (2016): Einführung in die Semantik. Grundlagen - Analysen - Theorien. Stuttgart: J.B. Metzler.

Pană Dindelegan; Gabriela (2012): Feminine singular pronouns with neutral value. In: Revue Roumaine de Linguistique LVII, 3, 249-261.

Pană Dindelegan, Gabriela (Hg.) (2013): The Gammar of Romanian. Oxford etc.: Oxford University Press.

Paranhos Zitterbart, Jussara (2013): Satztyp und Korrelat / Platzhalter / Bezugsausdruck. In: Meibauer, Jörg / Steinbach, Markus / Altmann, Hans (Hg.): Satztypen des Deutschen. Berlin/New York: de Gruyter, 602-626.

Paul, Hermann (1919): Deutsche Grammatik. Bd. 3. Teil IV: Syntax (Erste Hälfte). Halle a. S.: Niemeyer.

Reis, Marga (1997): Zum syntaktischen Status unselbständiger Verbzweit-Sätze. In: Dürscheid, Christa / Ramers, Karl Heinz / Schwarz, Monika (Hg.): Sprache im Fokus. Tübingen: Niemeyer, 121-144.

Schmid, Hans-Jörg (2000): English Abstract Nouns as Conceptual Shells. From Corpus to Cognition. Berlin / New York: Mouton de Gruyter.

Stiebels, Barbara (2002): Typologie des Argumentlinkings: Ökonomie und Expressivität. (Studia grammatica 54). Berlin: Akademie Verlag.

Vasiliu, Emanuel (1978): Preliminarii logice la semantica frazei. București: Editura științifică și enciclopedică.

Vendler, Zeno (1967): Linguistics and Philosophy. Ithaca, New York: Cornell University Press. 
Winter, Eugene (1992): The notion of unspecific versus specific as one way of analyzing the information of a fund-raising letter. In Mann, W.C. / Thompson, S.A. (Hg.): Discourse description: diverse linguistic analyses of a fund-raising text. Amsterdam / Philadelphia: Benjamins, 131-170.

Zafiu, Rodica (2004): Observații asupra anaforei în limba română actuală. In: Pană Dindelegan, Gabriela (Hg.): Tradiție și inovație în studiul limbii române. București: Editura Universității din București, 239-252.

Zifonun, Gisela (2001): Grammatik des Deutschen im europäischen Vergleich. Das Pronomen. Teil 1: Überblick und Personalpronomen. amades working paper 4/01. Mannheim: Institut für deutsche Sprache.

Zifonun, Gisela / Hoffmann, Ludger / Strecker, Bruno (1997): Grammatik der deutschen Sprache. 3 Bände. (Schriften des Instituts für Deutsche Sprache 7.1-7.3). Berlin / New York: de Gruyter. 


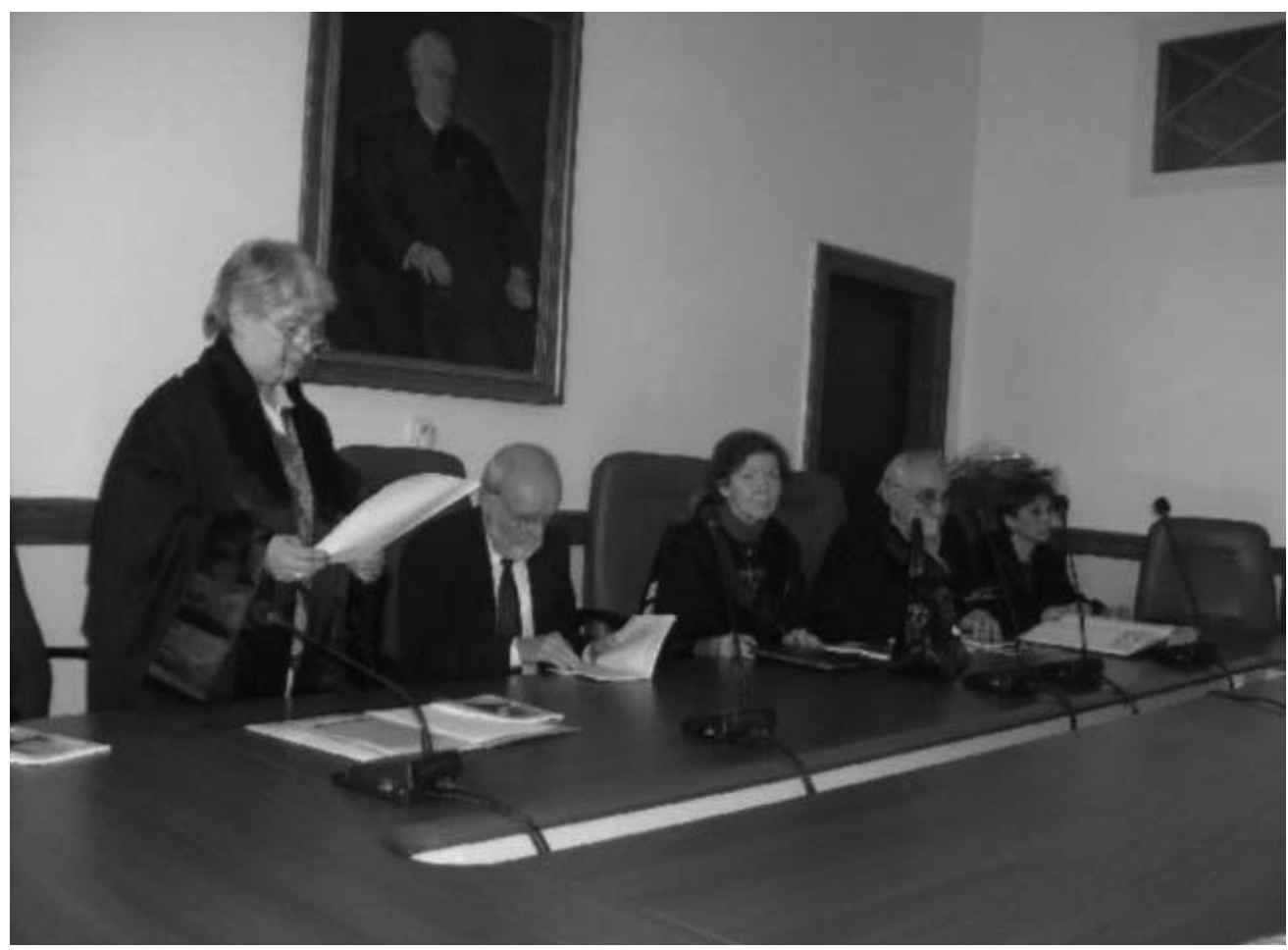

Verleihung der Ehrendoktorwürde an Prof. Dr. Ulrich Engel an der Universität Bukarest am 6.11.2008 (Prof. Speranta Stanescu, Prof. Alexandra Cornilescu [Dekanin] und Prof. George Gutu) 